www.jmscr.igmpublication.org

Index Copernicus Value: 79.54

ISSN (e)-2347-176x ISSN (p) 2455-0450

crossref DOI: https://dx.doi.org/10.18535/jmscr/v7i6.80

\title{
Operational Utility of Fractional Flow Reserve in Guiding the Decision for Coronary Revascularization in Patients of Coronary Artery Disease in Indian Scenario
}

\author{
Authors \\ Vikram Singh ${ }^{1}$, Sheikh Mohamad Tahir ${ }^{2}$, Jasmine Kang ${ }^{3}$, Shubana Ashraf ${ }^{4}$ \\ ${ }^{1}$ Consultant Cardiologist, Indus International Hospital, Mohali, Punjab \\ ${ }^{2}$ Consultant Cardiologist, Super Speciality Hospital, Government Medical College, J\&K, India \\ ${ }^{3}$ Consultant Gynaecologist, Indus International Hospital, Mohali, Punjab \\ ${ }^{4}$ Consultant Neurologist, Super Speciality Hospital, Government Medical College, J\&K, India
}

\begin{abstract}
Background: Atherosclerotic coronary heart disease (CHD) is the commonest non-communicable disease worldwide and contributes to more than 1.2 million myocardial infarctions and nearly 500,000 deaths each year. The aim of the study was to assess FFR in patients with coronary lesions of intermediate/uncertain severity after conventional CAG and derive revascularization decision thereby. To assess how often FFR changes revascularization decisions in such patients and to analyse inter observer variability in angiographic decision making between experienced operators in coronary lesions of intermediate/uncertain severity.

Methods: We enrolled 40 patients with coronary lesions of intermediateluncertain severity whose revascularization decision remained ambiguous as assessed by the primary operator after clinical and noninvasive data (TMT, DSE, stress MIBI) and coronary angiography.

Results: A total of 40 patients were enrolled for the study among which $79.3 \%$ were males and $20.7 \%$ females. The Mean age ( $\pm S D)$ in female group was $64.5 \pm 6.097$ years and that in male group was $59.3 \pm$ 11.02 years. Eight coronary lesions were found to be of intermediatel uncertain severity and were subjected to FFR. All lesions having FFR $\leq 0.8$ underwent intervention while none of the lesion having FFR value of $\geq$ 0.8 were intervened. So there was significant association found between FFR value and decision changed.

Conclusion: FFR provides a reliable and objective assessment of intermediateluncertain lesions leading to better revascularization decisions.

Keywords: Coronary Revascularization, coronary artery disease, Fractional Flow Reserve, FFR.
\end{abstract}

\section{Introduction}

Atherosclerotic coronary heart disease (CHD) is the commonest non-communicable disease worldwide and contributes to more than 1.2 million myocardial infarctions and nearly 500,000 deaths each year ${ }^{(1)}$. Globally $30 \%$ of the deaths can be attributed to cardiovascular disease, of which more than half are caused by CHD. The presence of myocardial ischemia is an important risk factor towards an adverse clinical outcome ${ }^{(2-}$ 4). Coronary revascularization by Percutaneous Coronary Interventions (PCI) or Coronary Artery 
Bypass Grafting can improve symptoms as well as enhance survival in properly selected patients. The presence of ischemia has emerged as a key predictor of improvement after coronary revascularization. Revascularization of stenotic coronary lesions that induce ischemia can improve a patient's functional status and outcome. For stenotic lesions that do not induce ischemia, the benefit of revascularization is uncertain ${ }^{(5-8)}$.

Coronary angiography is the standard technique for assessing the coronary arteries, but it has its limitations in determining the physiologic significance of coronary stenosis. In patients with chest pain and stenosis of moderate severity, as assessed by coronary angiography, evaluation and treatment are challenging as the angiographic findings may not correlate with functional severity. With the introduction of drug-eluting stents, the percentage of patients with multi-vessel coronary artery disease in whom percutaneous coronary intervention (PCI) is performed has increased $^{(9,10)}$.

Coronary angiography often underestimates or overestimates a lesion's functional severity. Consequent to the above, the intracoronary physiologic measurement of myocardial fractional flow reserve (FFR) was introduced and has proven to be a reliable method for determining the functional severity of coronary stenosis. Fractional flow reserve (FFR) is an index of the physiological significance of a coronary stenosis and is defined as the ratio of maximal hyperaemic blood flow in a stenotic artery to normal maximal flow $^{(14-16)}$.

FFR concept: FFR is a lesion-specific index of epicardial stenosis severity and represents the fraction of normal maximum flow that remains despite the stenosis. In the absence of stenosis, the driving pressure over the myocardium is 100 $\mathrm{mmHg}$ at maximum vasodilatation. The presence of stenosis, however, results in a hyperemic gradient of $40 \mathrm{mmHg}$, consequently, overall maximum driving pressure fall to only $60 \mathrm{mmHg}$. This implies that in the absence of stenosis, the maximum blood flow in this stenotic artery goes down to only $60 \%$ of normal. By definition, the FFR is 0.6. In other words, FFR is the ratio of maximum hyperaemic blood flow measured in the presence of a focal coronary stenosis to the normal hyperaemic blood flow in the same vessel in the absence of stenosis. It can be calculated as under:-

$$
\mathrm{FFR}=(\mathrm{Pd}-\mathrm{PV}) /(\mathrm{Pa}-\mathrm{Pv})=\mathrm{Pd} / \mathrm{Pa}
$$

Where $\mathrm{Pa}$ is the mean aortic pressure measured from the guiding catheter, $\mathrm{Pd}$ is the distal coronary pressure measured from the pressure-sensing guide wire, and $\mathrm{Pv}$ is the central venous pressure, all measured at maximum coronary hyperaemia. Since central venous pressure is close to zero, PV is negligible. Thus, FFR can easily be derived from the ratio of mean distal coronary artery pressure to aortic pressure during maximal hyperaemia. The theoretical value for FFR of a normal coronary artery is 1.0, regardless of vessel or the patient. The measurement of FFR is independent of changes in systemic blood pressure, heart rate, or myocardial contractility and is highly reproducible ${ }^{(20,21)}$. The role of FFR measurement is very useful in identifying patients with multi-vessel disease who might benefit from coronary revascularization. If acceptable physiologic assessment criteria are met for all the lesions, catheter based treatment or coronary bypass surgery can be safely deferred. For patients with multi-vessel coronary disease, it is important to know which particular lesion is physiologically significant and is responsible for reversible ischemia. With the help of FFR measurement, it is now possible to identify one or more culprit lesions in these types of patients.

Coronary angiograms are still frequently used as a cornerstone of decision making, despite the substantial discrepancy between the angiographic and functional severity of stenosis. Therefore, adjuvant technologies such as fractional flow reserve (FFR) and intravascular ultrasound (IVUS) are considered in daily practice to overcome the limitations of coronary angiography for diagnostic and interventional procedures. Therefore, adjuvant technologies such as 
fractional flow reserve (FFR) and intravascular ultrasound (IVUS) are considered in daily practice to overcome the limitations of coronary angiography for diagnostic and interventional procedure. In contrast to the benefits of percutaneous coronary intervention (PCI) in patients with unstable angina and myocardial infarction, the benefits of PCI in patients with stable angina are less clear ${ }^{(25,26)}$.

Nevertheless, recent advances in drug-eluting stents (DES) and adjuvant pharmacological agents may reduce the thresholds for revascularization therapy in the absence of firm evidence of objective ischemia. Thus, consideration is sometimes given to treating stenosis of intermediate degree without consideration of their functional significance. In addition, although most surgical recommendations for patients with multivessel coronary artery disease are to bypass all lesions with diameter stenosis of $>50 \%$ for complete revascularization, the patency rate of vein grafts on vessels with functionally insignificant proximal stenosis has been in question $^{(27,28)}$. The non-invasive tests of inducible ischemia (exercise treadmill test, stress nuclear myocardial perfusion imaging, and exercise stress echocardiography) are also limited by their poor sensitivity in localizing the lesions FFR has gained importance with guidelines recommending class I indication for lesions without objective evidence of ischemia. The visual functional mismatch would be maximal in smaller vessels and where there is a disproportion between the vessel size and myocardium supplied by it ${ }^{(29,30)}$.

Despite these studies there is a paucity of data on FFR from Indian patients. Our patients would probably benefit more because of their relatively smaller epicardial coronaries than Caucasians and the frequent occurrence of multi vessel, small vessel, and diffuse disease $\mathrm{e}^{(31,32)}$.

\section{Aim and Objectives}

1. To assess FFR in patients with coronary lesions of intermediate/uncertain severity after conventional $\mathrm{CAG}$ and derive revascularization decision thereby.

2. To assess how often FFR changes revascularization decisions in such patients

3. To analyze inter observer variability in angiographic decision making between experienced operators in coronary lesions of intermediate/uncertain severity.

\section{Material and Methods}

Study Design: Cohort Study.

Study period: January 2012-December 2015.

Study Institution: Batra Hospital and Medical Research Centre New Delhi.

Procedure: We enrolled 40 patients with coronary lesions of intermediate/uncertain severity whose revascularization decision remained ambiguous as assessed by the primary operator after clinical and non-invasive data (TMT, DSE, stress MIBI) and coronary angiography. All lesions with diagnostic uncertainty were subjected to FFR. Intracoronary bolus adenosine (80-100 $\mu \mathrm{g})$ was administered to induce maximum hyperaemia, and FFR was estimated with 0.014 inch sensor tipped Pressure Wire Certus TM (St. Jude Medical) attached with St Jude Medical Radi Analyser TM Xpress 12711 - SN- 3249. Coronary lesions were considered significant at FFR $\leq 0.8$. Decision for revascularization was recorded at 2 stages viz. Stage I (angiographic): after coronary angiography including all clinical, non-invasive and angiographic information, and Stage II (post FFR): After adding the information from FFR. We analysed the difference between Stage I and Stage II decision as a measure of the utility of FFR. The clinical and non-invasive data and coronary angiography CDs of these study patients were also shown retrospectively to two independent experienced cardiologists. They were blinded to FFR findings and actual treatment assignment. Their revascularization decisions were recorded and compared with that of primary operator to assess inter-observer variability and assess whether consensus decision making might 
be expected to reduce the need for objective functional testing by FFR. Those with primary percutaneous coronary intervention acute coronary syndrome with hemodynamic compromise.

Statistical Methods: The descriptive statistical analysis was done using mean or median with standard deviation (SD) for quantitative variables and categorical variables were presented in frequencies along with respective percentages. Pie chart and bar diagrams were made for graphical presentation of data. The statistical comparisons for quantitative variables were done using Student's t- test. The association between categorical variables was measured by Chi-square or Fisher's exact test. Scatter plots were made to visualize the linear relationship between the quantitative variables. Linear correlation was measured by Pearson's product moment correlation. The internal consistency among the consultants was assessed by intraclass correlation coefficient using two-way mixed effect model. All statistical analyses were performed by using SPSS software (Version 21, SPSS Inc, Chicago, IL, USA). A p value less than 0.05 was considered as statistically significant.

Ethical Issues: Investigator and supervisor were aware of the ethics in biomedical research policy and declaration of Helsinki revised in 2002. Keeping this in view, written informed consent of all participants was obtained before gathering any information. The study involved no ethical issues related to human or animal experimentation.

\section{Results}

A total of 40 patients were enrolled for the study among which $79.3 \%$ were males and $20.7 \%$ females. The Mean age ( \pm SD) in female group was $64.5 \pm 6.097$ years and that in male group was $59.3 \pm 11.02$ years. Eight coronary lesions were found to be of intermediate/ uncertain severity and were subjected to FFR. Table 1 shows that thirty seven out of 58 lesions $(68.8 \%)$ had diameter stenosis $<70 \%$ and $21(31.2 \%) \geq 70 \%$. Table 2 describes the comparison of lesions after FFR.
$70.7 \%$ of the total lesions were having FFR value of more than 0.8 and remaining $29.3 \%$ were $\leq 0.8$. All lesions having FFR $\leq 0.8$ underwent intervention while none of the lesion having FFR value of $\geq 0.8$ were intervened. So there was significant association found between FFR value and decision changed.

Decision to re-vascularize the vessel or to defer the procedure was changed for 12 lesions $(20.6 \%)$ based on an FFR cut-off value of $\leq 0.8$. Of these, 4 lesions $(33 \%)$ were $<70 \%$ stenosed and 8 were $\geq 70 \%(66.6 \%)$. In 8 cases FFR led to deferral of procedure and in 4 cases it led to intervention (3 PCI, $1 \mathrm{CABG}$ ). In other cases it served to validate the angiographic decision. The decision change was commonly in favour of deferral (8 out of 12 lesions) but led us to perform procedure in 4 cases as shown in Table 3.Scatter diagram (Fig.1) depicts the correlation between lesion percent and FFR value. The negative and significant correlation $(\mathrm{r}=-0.567 ; \mathrm{p}<0.01)$ exists between lesion percent and FFR value. It clearly indicated that as lesion percent increases the FFR value decreases.

Performance of FFR in 40 cases (58 lesions) with coronary lesions of intermediate/uncertain severity led to avoidance of 8 stents in nonsignificant lesions and permitted the additional treatment of 4 lesions that required intervention. This is expected to improve the clinical results and save cost although long term outcome and cost benefit were not part of the study. Interobserver variability of revascularization decision was assessed between three cardiologists (1 primary and 2 retrospective). The angiographic decisions were concordant in $75 \%$ cases with interclass correlation of 0.745 . In 14 lesions, there was difference of opinion between the three cardiologists. In these cases FFR confirmed the primary operator's decision in 10 but differed in 4 out of 14 lesions. (Table $4 \&$ Figure 2). The three cardiologists had unanimous angiographic opinion in 44 out of 58 lesions, largely eliminating observer bias or error in these lesions. Despite this FFR decision differed from the unanimous 
angiographic opinion in 8 out of 44 lesions $(18.2 \%)$. The highest mean $( \pm \mathrm{SD})$ score of lesion percent was given by consultant $3(62.59 \pm 12.78)$ followed by consultant1 $(61.88 \pm 12.86)$ and consultant2 (60.52 \pm 14.32$)$. The linear product moment correlation was positive and statistically significant $(\mathrm{p}<0.01)$. The internal consistency or agreement among the consultants was estimated as $74.5(95 \% \mathrm{CI}=64 \%-83 \%)$. It indicated the fair amount of agreement among the consultants.

Table 1: Comparison of \% diameter stenosis of coronary lesions and decision after angiography

\begin{tabular}{|c|c|c|c|c|c|c|c|c|}
\hline \multirow[t]{3}{*}{ Lesion \% } & \multicolumn{4}{|c|}{ Decision after angiography } & \multirow{2}{*}{\multicolumn{2}{|c|}{ Total }} & \multirow[t]{3}{*}{ Chi-square $^{\#}$} & \multirow[t]{3}{*}{ p value } \\
\hline & \multicolumn{2}{|c|}{ Defer } & \multicolumn{2}{|c|}{ Perform } & & & & \\
\hline & $\mathbf{n}$ & $\%$ & $\mathbf{n}$ & $\%$ & $\mathbf{n}$ & $\%$ & & \\
\hline$<70$ & 35 & 100.0 & 2 & 8.7 & 37 & 63.8 & \multirow[t]{3}{*}{50.09} & \multirow[t]{3}{*}{$0.000 * * *$} \\
\hline$>=70$ & 0 & 0 & 21 & 91.3 & 21 & 36.2 & & \\
\hline Total & 35 & 100.0 & 23 & 100.0 & 58 & 100.0 & & \\
\hline
\end{tabular}

Table No 2 Comparison of lesions after FFR

\begin{tabular}{|l|c|c|c|c|c|c|}
\hline \multirow{2}{*}{ FFR value } & \multicolumn{4}{|c|}{ Decision after FFR } & \multicolumn{2}{c|}{ Total } \\
\cline { 2 - 7 } & \multicolumn{2}{|c|}{ Defer } & \multicolumn{2}{c|}{ Perform } & \multicolumn{2}{c|}{ Torn } \\
\cline { 2 - 7 } & $\mathbf{n}$ & $\mathbf{\%}$ & $\mathbf{n}$ & $\mathbf{n}$ & $\mathbf{\%}$ \\
\hline$<=0.8$ & 0 & 0 & 17 & 100 & 17 & 29.3 \\
\hline$>0.8$ & 41 & 100 & 0 & 0 & 41 & 70.7 \\
\hline Total & 41 & 100.0 & 17 & 100.0 & 58 & 100.0 \\
\hline
\end{tabular}

Table 3: Association between lesion percent and final decision after FFR

\begin{tabular}{|l|c|c|c|c|c|c|c|c|}
\hline \multirow{3}{*}{ Lesion \% } & \multicolumn{4}{|c|}{ Final Decision } & \multicolumn{2}{|c|}{ Total } & \multirow{2}{*}{ Chi-square } & \multirow{2}{*}{ p value } \\
\cline { 2 - 7 } & Decision changed & \multicolumn{2}{|c|}{ Decision not changed } & \multicolumn{2}{c|}{} \\
\cline { 2 - 7 } & $\mathbf{n}$ & $\mathbf{\%}$ & $\mathbf{n}$ & $\mathbf{n}$ & $\mathbf{n}$ & $\boldsymbol{\%}$ & & \\
\hline$<70$ & 4 & 33.3 & 33 & 71.7 & 37 & 63.8 & \multirow{2}{*}{6.08} & \multirow{2}{*}{$\mathbf{0 . 0 2 0} *$} \\
\hline$>=70$ & 8 & 66.7 & 13 & 28.3 & 21 & 36.2 & & \\
\hline Total & 12 & 100.0 & 46 & 100.0 & 58 & 100.0 & & \\
\hline
\end{tabular}

Figure 1: Correlation between FFR value and \% of lesion

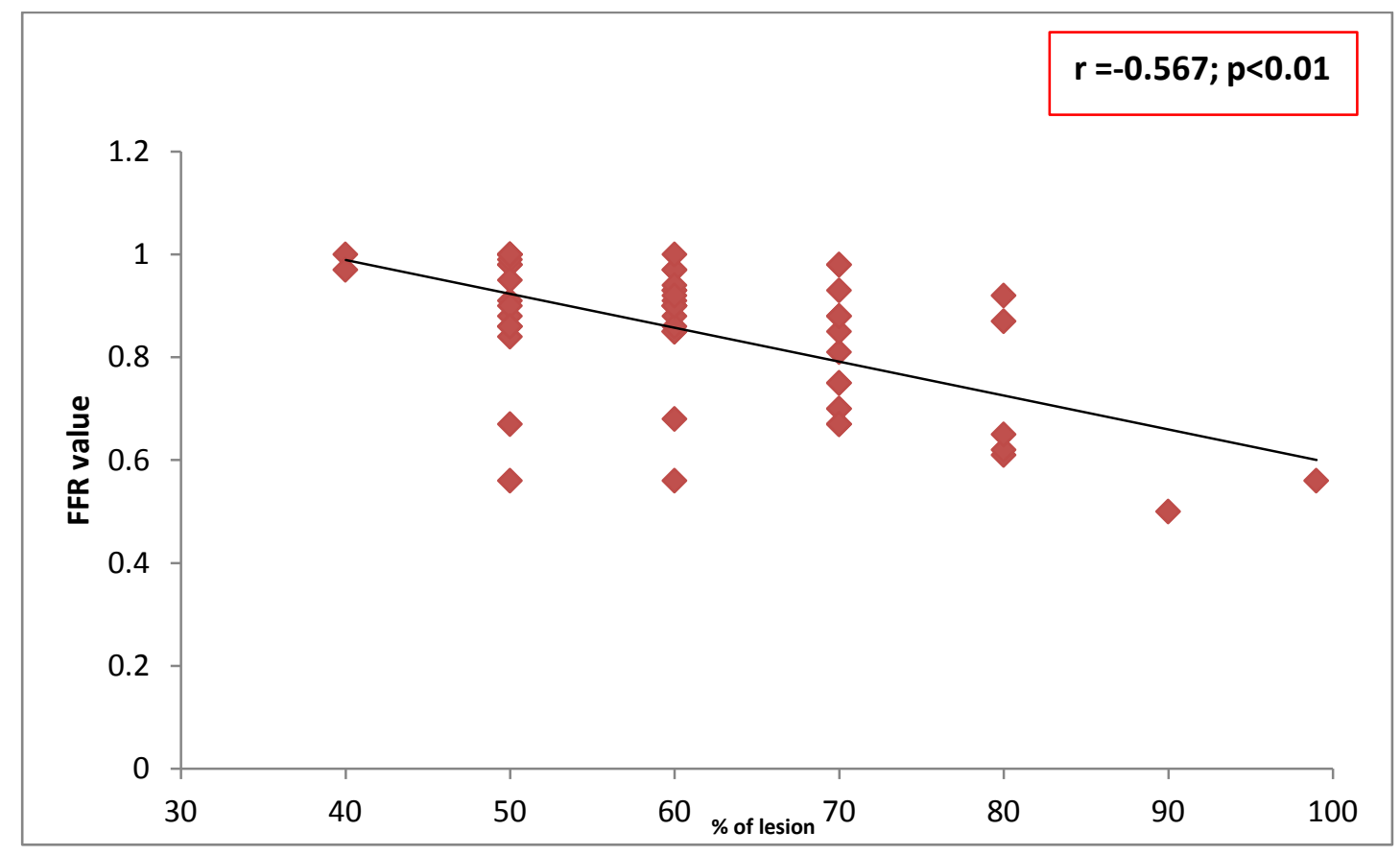


Figure 2: Inter consultant's correlation matrix for assessing lesion percent (\%)

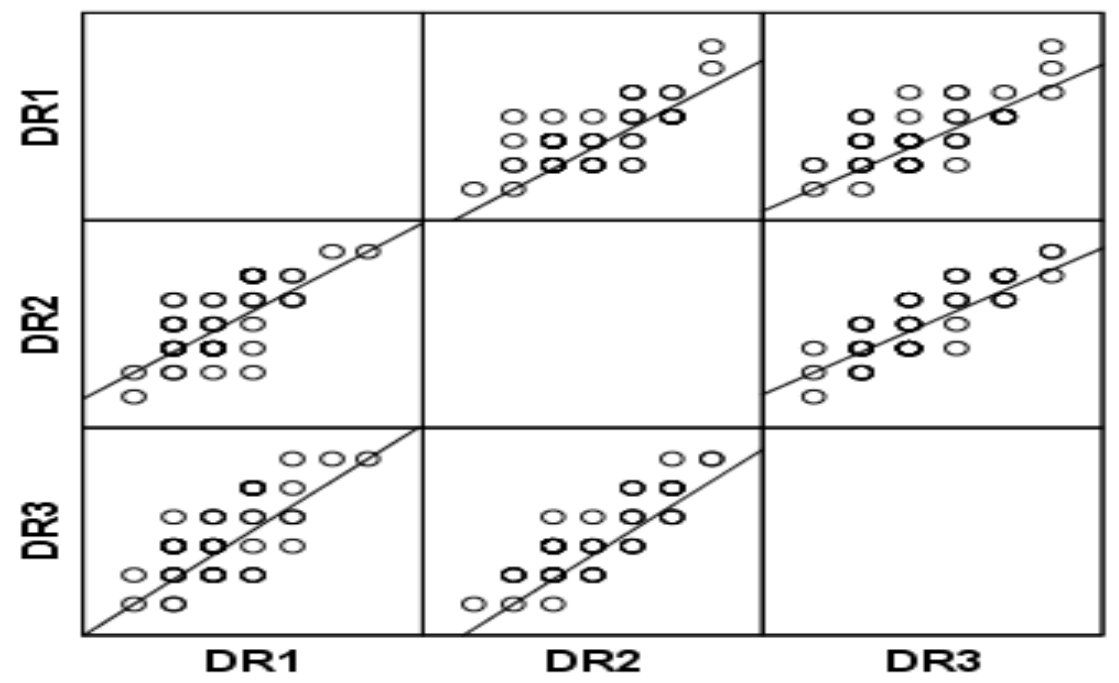

Table 4: Intra class correlation analysis among the consultants

\begin{tabular}{|c|c|c|c|c|c|c|c|c|}
\hline \multirow{2}{*}{$\begin{array}{l}\text { Type of } \\
\text { consultant }\end{array}$} & \multicolumn{3}{|c|}{ Inter-Item Correlation Matrix } & \multirow{2}{*}{$\begin{array}{l}\text { Intraclass } \\
\text { correlation }\end{array}$} & \multicolumn{2}{|c|}{ 95\% C.I. } & \multirow{2}{*}{$\begin{array}{c}\mathbf{F} \\
\text { value }\end{array}$} & \multirow[b]{2}{*}{ p value } \\
\hline & DR1 & DR2 & DR3 & & $\begin{array}{l}\text { Lower } \\
\text { Bound }\end{array}$ & $\begin{array}{l}\text { Upper } \\
\text { Bound }\end{array}$ & & \\
\hline DR1 & 1 & & & \multirow{5}{*}{0.745} & \multirow{5}{*}{0.64} & \multirow{5}{*}{0.83} & \multirow{5}{*}{9.78} & \multirow{5}{*}{$0.000 * * *$} \\
\hline DR2 & $0.71 * *$ & 1 & & & & & & \\
\hline DR3 & $0.69 * *$ & $0.84 * *$ & 1 & & & & & \\
\hline Mean & 61.88 & 60.52 & 62.59 & & & & & \\
\hline $\mathrm{SD}$ & 11.86 & 14.32 & 12.78 & & & & & \\
\hline
\end{tabular}

\section{Discussion}

FFR provides a reliable and objective assessment of intermediate/uncertain lesions leading to better revascularization decisions. The utility and value of FFR assessment in determining the hemodynamic significance of coronary vessels has been demonstrated and validated in a series of high-quality scientific studies, including Fractional Flow Reserve to Determine the Appropriateness of Angioplasty in Moderate Coronary Stenosis (DEFER), ${ }^{(35)}$ Fractional Flow Reserve (FFR) vs. Angiography in Multi-vessel Evaluation (FAME) ${ }^{(33)}$ and FAME-2 ${ }^{(34)}$. However, the utilization of FFR measurement in practice will depend on how often it changes the angiogram- derived assessment of the distribution of significant $\mathrm{CAD}$ and hence revascularization decision.

Our plan was to assess how often FFR changes revascularization decision in patients with coronary lesions of intermediate/uncertain severity after performing conventional angiography and also to assess inter observer variability in angiographic decision making between experienced operators in such lesions. Our study has shown that FFR measurement leads to a change in management plan in $20.6 \%$ of in intermediate/uncertain lesions based on an FFR cut-off value of $\leq 0.8$. This observation is consistent with the recently published FIND study by Sengottuvelu G, et al. ${ }^{(37)}$ although the percentage of decision change in their paper was $40 \%$ which is higher than in our study.

Inter-observer variability of revascularization decisions between three senior cardiologists was also studied by us. Decisions were concordant in $75 \%$ cases with interclass correlation of 0.745 , although in 14 lesions, there was difference of opinion between the three. This again points to the need for objective evaluation in intermediate/ uncertain lesions. 
Interobserver variability has also been studied by Lindstaedt $\mathrm{M}$, et al. ${ }^{(51)}$ They compared the accuracy of visual angiographic assessment of intermediate LMCA stenosis by experienced interventional cardiologists with functional assessment by FFR in a patient population with excellent long-term outcome after deferral of surgery on the basis of FFR measurements. Inter observer concordance was found in only $46 \%$ which was lower than our study.

In the RIPCORD STUDY done by Nick Curzen, et al. $^{(38)}$ the protocol allowed the second cardiologist to perform FFR according to his assessment of the vessels. Also FFR was allowed in all lesions including those $>70 \%$. The discordance between the two management decisions in this trial was $28 \%$. This observation is consistent with our study where 21 lesions of intermediate/uncertain severity $(31.2 \%)$ had diameter stenosis $\geq 70 \%$. In our study, out of 12 lesions in which decision was changed, 8 were $\geq 70 \%(66.6 \%)$ and $4<70 \%$ $(33 \%)$. Hence coronary angiography can both overestimate and underestimate the functional severity of a lesion.

The decision change in our study was commonly in favour of deferral (8 out of 12 lesions) but led us to perform procedure in 4 cases (3 PCI, 1 CABG). Performance of FFR in 40 cases (58 lesions) with coronary lesions of intermediate/ uncertain severity led to avoidance of 8 stents in non-significant lesions and permitted the additional treatment of 4 lesions that required intervention. This is expected to improve the clinical results and save cost although long term outcome and cost benefit were not part of the study.

So there is definite advantage to use FFR as an adjunct to diagnostic angiography in decision making. Furthermore, the potential value of FFR in the precise identification of hemodynamically significant coronary lesions is likely to be as high for those not already labelled as requiring PCI, as was the case in DEFER, ${ }^{(35)}$ FAME,${ }^{(33)}$ and FAME$2^{(34)}$. Also the data from RIPCORD ${ }^{(38)}$ indicate that routine application of FFR at the stage of diagnostic angiography would have a profound effect on the assessment and management of those patients in whom angiogram alone suggests medical therapy alone or CABG would be the optimal treatment plan. The potential value extends toward informing when not to revascularize as well as which revascularization modality is optimal for an individual patient.

\section{Conclusions}

FFR provides a reliable and objective assessment of intermediate/uncertain lesions leading to better revascularization decisions. There is significant inter-observer variability between cardiologists when assessing intermediate/uncertain lesions and FFR changes decisions even when the subjective decision is unanimous. In our experience of intermediate / uncertain coronary lesions FFR changed the treatment assignment in $20.6 \%$ leading to the avoidance of stents in 8 lesions and appropriate revascularization in 4 additional lesions. Angiographic decisions of experienced operators differed in $25 \%$ of intermediate/ uncertain lesions. FFR also changed the treatment assignment in $18.2 \%$ of lesions where the angiographic assessment was unanimous, further attesting to the need for objective functional testing in these cases.

\section{Limitations of our study}

1) Small sample size.

2) FFR value was not validated with other investigational modalities like SPECT, IVUS or OCT.

3) Lack of long-term follow-up which would correlate the final FFR influenced decision with clinical outcomes. However this has been studied in several larger studies.

4) The performance of FFR with bolus doses of adenosine may be inferior to continuous intravenous infusion especially in left main and serial stenosis. 


\section{Recommendations}

1) FFR should be used when there is apparent discordance between lesion severity, location of ischemia by non-invasive methods and symptoms; FFR provides valuable data in clinical decision making.

2) Instead of relying solely on angiographic criteria of severity when there is no stress test present, or stress test of anatomy results are discordant FFR can be the final arbiter irrespective of lesion severity.

3) FFR can be used to evaluate the serial stenosis to guide the strategy for determining which lesions should be revascularise and which should be manage medically.

4) Physiologically guided PCI using FFR is safe and cost effective and reduces the number of stents required to treat patients with multi-vessel CAD.

5) FFR is safe and can be done in the same sitting.

\section{Conflict of Interest: None}

\section{Source of Funding: None}

\section{References}

1. Lloyd-Jones, Adams R, Carnation M, et al. Heart Disease and Stroke Statistics--2009 update. A report from the American Heart Association Statistics Committee and Stroke Statistics Subcommittee.2009; 119: $480-486$.

2. Baller GA, Zaret BL. Contributions of Nuclear Cardiology to diagnosis and prognosis of patients with Coronary Artery Disease. Circulation 2000; 101:1465-78.

3. Shaw LJ, Iskandrian AE. Prognostic value of Gated Myocardial Perfusion SPECT. Journal of Nuclear CardiologyMarch-April 2004; 11:171-85.

4. Shaw LJ, Berman DS, Maron DJ, et al. Optimal Medical Therapy with or without Percutaneous Coronary Intervention to reduce ischemic burden. Circulation 2008; 117:1283-9

5. Davies RF, Goldberg AD, Forman S, et al. Asymptomatic Cardiac Ischemia Pilot (ACIP) Study Two-Year Follow-up. Outcomes of patients randomized to initial strategies of Medical Therapy versus Revascularization. Circulation 1997; 95:2037-43.

6. Erne P, Schoenenberger AW, Burckhardt D, et al. Effects of Percutaneous Coronary Interventions in silent ischemia after Myocardial Infarction: The SWISSI II Randomized Controlled Trial. JAMA. 2007;297(18):1985-1991

7. Boden WE, O Rourke RA, Teo KK, et al. Optimal Medical Therapy with or without PCI for Stable Coronary Disease. N Engl J Med 2007; 356:1503-16.

8. Pijls NH, Van Schaardenburgh P, Manoharan G, et al. Percutaneous Coronary Intervention of functionally nonsignificant stenosis. 5 - Year follow-up of the DEFER Study. J Am CollCardiol 2007; 49:2105-11.

9. Moses JW, Stone GW, Nikosky E, et al. Drug-Eluting Stents in the treatment of intermediate lesions. Pooled analysis from four randomized trials $\mathrm{J}$ Am CollCardiol 2006; 47:2164-71.

10. Ong AT, Van Domburg RT, Aoki J, et al. Sirolimus-Eluting Stents remain superior to Bare-Metal Stents at two years: Medium-term results from the RapamycinEluting Stent Evaluated at Rotterdam Cardiology Hospital (RESEARCH) Registry. J Am CollCardiol 2006; 47:1356-60.

11. Pijls NH, Van Gelder B, Van der Voort P, et al. Fractional Flow Reserve: A useful index to evaluate the influence of an Epicardial Coronary Stenosis on Myocardial Blood Flow. Circulation. 1995; 92:3183-3193. 
12. De Bruyne, B, Pijls NH, VanGelder B, et al. Relation between Myocardial Fractional Flow Reserve calculated from Coronary Pressure Measurements and exercise-induced Myocardial Ischemia. Circulation. 1995; 92:39-46.

13. Pijls NH, De Bruyne B, Peels K, et al. Measurement of Fractional Flow Reserve to assess the functional severity of Coronary Artery Stenosis. N Engl. J Med 1996; 334:1703-8.

14. De Bruyne B, Bartunek J, Sys Su, et al. Relation between Myocardial Fractional Flow Reserve calculated from Coronary Pressure Measurements and exerciseinduced Myocardial Ischemia. Circulation 199; 92:39-46.

15. De Bruyne B, Bartunek J, Sys S, et al. Simultaneous Coronary Pressure and Flow Velocity measurements in humans. Feasibility, reproducibility, and hemodynamic dependence of Coronary Flow Velocity Reserve, Hyperemia Flow versus Pressure Slope Index and Fractional Flow Reserve. Circulation 1996; 94:1842-9.

16. Frye RL, August P, Brooks MM, et al. A randomized trial of therapies for type 2 Diabetes and Coronary Artery Disease. N Engl J Med. 2009; 360:2503-2515.

17. Boden WE, O Rourke RA, Teo KK, Hartigan PM, et al. Optimal Medical Therapy with or without PCI for Stable Coronary Disease. N Engl J Med. 2007; 356:1503-1516.

18. Moses JW, Stone GW, Nikolsky E, et al. Drug-Eluting Stents in the treatment of intermediate lesions: Pooled analysis from four randomized trials. J Am CollCardiol. 2006; 47:2164-2171.

19. Botman CJ, Schonberger J, Koolen S et al: Does stenosis severity of Native Vessels influence bypass graft patency? A prospective Fractional Flow Reserveguided study. Ann Thorac Surg. 2007; 83:2093-2097.
20. Wijns W, Kolh P, Danchin N et al. Guidelines on Myocardial Revascularization: The Task Force on Myocardial Revascularization of the European Society of Cardiology (ESC) and the European Association for CardioThoracic Surgery (EACTS). Eur Heart J 2010; 31:2501-2555.

21. Kushner FG, Hand M, Smith SC Jr, et al. 2009 Focused Updates: ACC/AHA guidelines for the management of patients with ST-Elevation Myocardial Infarction (updating the 2004 guideline and 2007 focused update) and ACC/AHA/SCAI guidelines on Percutaneous Coronary Intervention (updating the 2005 guideline and 2007 focused update): A report of the American College of Cardiology Foundation/American Heart Association Task Force on Practice Guidelines. Circulation 2009; 120:2271-2306.

22. Upendra K, Vineet B: Perspective on Coronary Interventions and Cardiac Surgeries in India. Indian J Med Res 2010; 132:543-548.

23. Varghese PJ, Arumugam B, Cherian K, et al. Atheromatous plaque reflects serum total Cholesterol levels: A comparative morphologic study of Endarterectomy Coronary Atherosclerotic Plaques removed from patients from the southern part of India and Caucasians from Ottawa, Canada. Clin Cadiol 1998; 2:135-340.

24. Bernard De Bruyne, William F. Fearon, Nico H.J. Pijls, et al. Fractional Flow Reserve-Guided PCI for Stable Coronary Artery Disease. FAME 2 Trial Investigators. N Engl J Med. September 1, 2014.

25. Pim A.L. Tonino, Bernard De Bruyne, Nico H.J. Pijls, et al. Fractional Flow Reserve versus Angiography for Guiding Percutaneous Coronary Intervention. N Engl J Med 2009; 360:213-224January 15, 2009. 
26. Bernard De Bruyne, Nico H.J. Pijls, BinduKalesan, et al. Fractional Flow Reserve-Guided PCI versus Medical Therapy in Stable Coronary Disease. N Engl J Med 2012; 367:991-1001September 13, 2012.

27. Leslee J. Shaw, Daniel S. Berman, David J. Maron, et al. Optimal Medical Therapy with or without Percutaneous Coronary Intervention to reduce ischemic burden. Circulation. 2008; 117: 1283-129.

28. Sun LJ, Mi L, Cui M, et al. Association between Fractional Flow Reserve and Quantitative Coronary Angiography Parameters in Intermediate Coronary Artery Stenosis. Zhonghua Xin Xue Guan Bing ZaZhi. 2012 Sep; 40:742-6.

29. G. Sengottuvelu, Babu Chakravarthy, Ravindran Rajendran, et al. Clinical usefulness and cost effectiveness of Fractional Flow Reserve among Indian patients. Catheterization and Cardiovascular Interventions 00:00-00 (2014). 